\title{
A Deterministic Model of Path Memory Dependent Inter-Nucleon Forces
}

\author{
Bei G* \\ Secondary School Teacher, IISS Charles Darwin, Italy
}

*Corresponding author: Gianpaolo Bei, Secondary School Teacher, IISS Charles Darwin, Via Tuscolana 388, Rome, Italy, Tel: +39 328 6779231; Email: capo.gian@libero.it

\section{Research Article}

Volume 5 Issue 1

Received Date: June 17, 2021

Published Date: June 30, 2021

DOI: $10.23880 / p s b j-16000178$

\section{Abstract}

We discuss a classical deterministic model of internucleon forces assuming that their irreversible dynamics is not described by instantaneous forces but by electrodynamic path dependent forces. We propose to describe the hidden dynamics of single accelerated and deformable nucleons by a hidden nuclear electromagnetic pilot wave in analogy with recent discussed hydrodynamic pilot wave models. We assume that the hidden dynamics is non- Hamiltonian and suggest to interpret nuclear statistics to be caused by hidden time dependent ground state and binding energy. We introduce single nucleon scalar potential describing its microscopic dynamic state as a quasi-particle state and we couple it in a self-consistent way to hidden nuclear vacuum index, generalizing a previous model of the author. We discuss an iterative method to find approximate solutions of our model. We introduce a nuclear Bernoulli principle and nuclear electromagnetic vector potential which we exploit to deduce a condition of dynamic equilibrium between nucleon repulsive forces and nucleon cohesive superficial forces. Finally we recapitulate our general critiques to standard description of nuclear dynamics and discuss some possible non Maxwell formulation of electrodynamic forces for accelerated systems. We speculate that new classical electrodynamical models could explain in a coherent way interesting phenomena, compatible and predicted by our model, such time dependent nucleon dipoles, delayed nucleon emissions and delayed electromagnetic emissions.

Keywords: Dissipative Nuclear Dynamics; Memory Dependent Nuclear Forces; Nuclear Pilot Wave; Hidden Nuclear Vacuum Index

\section{Introduction}

Since the discovery of nuclear fission [1,2], the cause of the instability of heavy nuclei was attributed to the strong Coulomb repulsion between the protons bound to them. Anyway this reasonable hypothesis was not clearly observed in experiments but only indirectly deduced by interpretations of ensemble averages data. Therefore the existence of microscopic Coulomb like forces were inferred applying classical concepts of potential and kinetic energies and the formalism of Quantum Mechanics.
The great success obtained by the first model of nuclear fission [3] made unnecessary to critique the contradictory hypothesis on which it was developed. In particular we think it has to be asked why we can talk of Coulombian forces and kinetic energies. Anyway we cannot attribute any physical meaning in to the associated classical notion of velocity and acceleration, since they are "banned" by the standard Copenhagen interpretation of Quantum Mechanics [4]; consequently, even the supposed constant and stationary proton charge is not so strongly experimentally justified (as far as we know no direct experiment confirms this hypothesis 
and excludes the possibility of velocity and acceleration dependent nucleon charges).

Therefore, since the thirties, till now there has been not a great debate on contradictory use of Schrodinger equation and the Born interpretation of its quantum wave function. In fact even in the very recent interesting theoretical and experimental investigations on nuclear fission [5-8] and nuclear energy states it was accepted the standard framework based on the assumption that the nucleus and the nucleons have static energy states and properties; in fact in these papers were adapted classical concepts without questioning the contradictory use of transition probabilities and stationary cross sections to describe the diffusion processes of the extended accelerated shape variable nucleons and fission fragments.

We want to remark that another contradictory assumption in nuclear physics is the use of Hamiltonian dynamics for describing nucleon time evolution, while it is well known experimentally the irreversibility nature of fission dynamics and more generally of nuclear reactions [9]. Consequently we find inadequate and incomplete the standard description of scattering processes calculated on quantum wave functions belonging to the infinite dimensional Hilbert space.

On the contrary important nuclear processes such as neutron capture are described by a different mathematical framework developed by Gamow to describe nuclear capture processes [10] which was recently generalized making dependent on a hidden vacuum refraction index [11]. The formalism of Gamow kets was not endorsed by the Copenhagen standard interpretation since, we think, its dynamics is irreducible time asymmetric. This irreversible nature of nuclear dynamics may justify the lack of interest of the scientific community on a realistic dynamic formulation of a theory of decaying states and the absence of an analogue of Bell theorem for quantum unstable states (even if it could have helped to reformulate as real space-time process the quantum wave collapse hypothesis).

Consequently we propose an approach which deny the existence of individual nucleon stationary ground state and time independent nuclear binding energy; therefore we expect that, we must look for hidden time dependent nuclear decay rates and half-lives, generalizing Gamow decay rates [12] since the standard ones are just emergent values of ensemble averages. Our model assumes that nuclear forces can be explained by generalized Coulombian potentials whose charge could have hidden time dependence; our aim is to give a realistic microscopic description of nucleon dynamics.
We will not use, as in the standard approaches conventional nuclear quantum numbers, such as charge, spin and isospin, since we think they are not intrinsic quantities but are the byproduct of statistical conservation laws. On the contrary we conceive them as emergent properties of hidden space time dependent vacuum fluctuations. Therefore we propose a deterministic model of internucleon dynamics which it is inspired by an analogy with recent studies and experiments on hydrodynamic pilot waves [13], [14] and on a time asymmetric generalization of self-consistent model of nuclear reactions proposed recently by the author [15]. We conceive each nucleon as a pulsating shape variable droplet interacting in a fast oscillating medium, the polarized nuclear vacuum, described by the hidden variable light speed; the Bjerknes acoustic like forces [14] on the nucleon are path dependent and described by

$$
\vec{F}_{\tau}=\int_{t-\tau}^{t} \nabla P \dot{V} d t^{\prime}
$$

with $P$ the hydrodynamic like nuclear pressure, $\mathrm{V}$ the pulsating volume of the nucleon droplet and $\tau$ the duration of the interaction with the nucleus that could be approximated by

$$
\tau=\frac{h}{m_{0} c^{2}}
$$

with $m_{0}$ the nucleon rest mass.

We will describe nucleon dynamics by a Bernoulli fluidodynamic equation and we will assume that the ensemble averaged internucleon forces are caused by hidden path dependent forces on single nucleons.

We suggest that each stationary conventional nucleon potential, such as for example the spin orbit one or the Yukawa one, could be recovered by averages on the hidden variable of vacuum indexes.

We expect that anomalous experimental observations of phenomena such as delayed neutron emissions and gamma ray emissions could be explained by the model which we will expose in the next paragraph. We wish to outline that our tentative model, even if it is based on a path memory dynamic framework, shares the same deterministic approach , proposed recently by some authors, which is aimed to a dynamical formulation of vacuum polarization [16-18]; anyway, differently from these papers, our proposal tries to go beyond the standard quantum mechanics concepts of stationary ground states and excited individual quantum states and stationary cross sections on which it is based the great success of the statistical predictions of the standard approach. Therefore we hope that our path dependent 
microscopic dynamics of accelerated pulsating nucleons will stimulate new interest for the search of deterministic formulations of quantum mechanics of single systems.

In fact, we think, that notwithstanding the modern debate on Bell's Theorem seems to prefer the Copenhaghen interpretation of quantum mechanics $[19,20]$ quantum nuclear states and therefore atomic stable states are not completely and correctly described by Schrodinger equations and its stationary eigenfunctions defined by conserved discretized quantum numbers.

\section{Model}

As we explain in the introduction, our self-consistent model is a time asymmetric generalization of a previous author's model [15]. Our aim is to elaborate a classical electrodynamical framework that we believe could be useful to describe the microscopic dynamic of a single deformable nucleon interacting with an accelerated nucleus, conceiving the interaction in a similar way to that one of an electron quasi-bound to an atom.

Our proposal is based on the hypothesis that this nucleon bound states have an hidden time dependence and that the statistic of nuclear reaction is caused by an hidden time dependence of the nuclear ground states. We want to remark that the dynamic of interacting nucleons cannot be described, we think, by classical Maxwell Theory since it concerns accelerating and pulsating extended systems. Our point of view is that it's necessary to reframe nuclear forces exploiting old theories as Weber electrodynamics [21] or a recent Mashhoon's proposal of a classical non-local electrodynamics theory [22].

We start by observing that nucleon electric dipoles may depend on time directly or indirectly (if they depend on velocity or angular momentum); in both cases we can introduce the following path dependent force between nucleons

$$
\overrightarrow{F_{12}}=\int_{0}^{\tau} \frac{\overrightarrow{d_{1}} \times \overrightarrow{d_{2}}\left(t-t^{\prime}\right)}{4 \pi \in\left(\frac{c}{n}\right)^{2} r_{12}^{2}\left(t-t^{\prime}\right)} d t^{\prime}
$$

with $d$ the nucleon electric dipoles, $\varepsilon$ the dielectric constant and $n$ the index of refraction of the polarized nuclear vacuum. This path dependent force depends obviously on the detailed electric dipoles time dependent law, that is at the moment unknown; our aim is to develop in the next future a more accurate model which will deduce this hidden time dependence. Anyway we have developed a simplified framework to describe inter nucleon forces introducing a Klein-Gordon like equation in a dielectric medium (that is the polarized vacuum) with a dissipative term for the scalar nuclear electromagnetic potential

$$
\left(\nabla^{2}-\frac{n^{2}}{c^{2}} \frac{\partial^{2}}{\partial t^{2}}\right) \phi_{n}-\beta \frac{\partial \phi_{n}}{\partial t}=\frac{E_{\text {Nucleon }}^{2}(n)}{c^{2} \hbar^{2}} \phi_{n}
$$

where $\beta$ is a phenomenological constant and the nucleon binding energy comparing in the second member of the equation describes the quasi-stationary nucleon state dependent on the vacuum index $n$

$$
E_{\text {Nucleon }}(n)=\frac{m_{0} c^{2}}{n^{2}}-m_{0} c^{2}
$$

The scalar potential is coupled in a self-consistent way with the nuclear vacuum index by the following formula

$$
n=1+\frac{\alpha \phi_{n}}{m_{0} c^{2}}\left(\frac{\Delta \vec{L}^{2}}{\hbar^{2}}\right)(6)
$$

with $\alpha$ a phenomenological constant dependent on the nucleons number of the nucleus and the temperature and $m_{0}$ is the rest mass of the nucleus and $\Delta \vec{L}^{2}$ the difference between the squared angular momentum of the nucleon and the nucleus (we modify a similar formula of our previous model since the angular momentum influences the shape dynamics of the nucleus [7]).

We wish to outline that the solutions of this self-consistent system of equation can be found by approximation using an iterative method; it consists in introducing at the second member of equation (4) some trial potentials (for example a Yukawa one) and then finding the first approximation solving the first member of the same equation. Then we can substitute it in the second member of equations (6) and (5) to go to the second order approximation, inserting these variables in the first member of equation (4). We expect that a power expansion in $\alpha$ might converge to the true solution.

We shall try to apply our self-consistent model to describe the dynamic equilibrium between repulsive forces (a kind of vacuum friction forces) and attractive forces of the nucleon interacting with the nucleus

$$
\frac{\alpha}{\tau} \int_{t-\tau}^{t}(-\nabla \phi)(Z q) d t^{\prime}+\int_{t-\tau}^{t} \nabla P \dot{V} d t^{\prime}=\frac{n}{c \tau} \Delta K_{t o t}+\frac{n}{c \tau} \Delta E_{r a d}(7)
$$

with $\tau$ the interaction time of the nucleon with the nucleus (it can be approximated using the standard Gamow formula of decay rate), $q$ is the proton electric charge, $Z$ is the protons number of the nucleus, $V$ is the Volume of the pulsating nucleon, is the predicted variation of the Kinetic energy of the nucleon plus the nucleon and is the experimentally observed difference between the electromagnetic radiation emitted and absorbed during the nuclear process.

The previous formula depends on two hidden variables; the first one is the electromagnetic pressure of the dynamic 
polarized vacuum and we suggest could be defined by the following equation

$$
P=\frac{\in \dot{\vec{A}}^{2}}{8 \pi}(8)
$$

where $\vec{A}$ is the electromagnetic nuclear vector potential which is coupled to the scalar potential by the standard Lorentz gauge condition (inserting the variable light speed of the polarized nuclear medium) .

In this way it remains just to determine the volume oscillations of the pulsating nuclear droplet that, we suggest, could be obtained assuming that the liquid nucleus satisfy the following Bernoulli nuclear principle.

$$
P+\frac{1}{2} \rho \vec{v}^{2}+\phi=\gamma
$$

where $\rho$ is the nucleon mass density defined by

$$
\rho=\frac{E_{\text {Nucleon }}}{c^{2} V}(10)
$$

$\gamma$ is a constant dependent on the necleus chosen and $\vec{v}$ is the velocity of the polarized irrotational liquid nucleus defined by the following formula

$$
\vec{v}=D \nabla \phi
$$

with $D$ a diffusion constant to be found experimentally.

The last three equations allow to determine the hidden variable $\mathrm{V}$ at the first member of equation (7). The natural interpretation of the scalar potential in formula (10) is a nuclear electromagnetic pilot wave which supports the liquid model of nucleon interaction, in analogy with hydrodynamic pilot wave models [13]. We note that we can add a stability condition on the nucleus assuming that the temporal derivative of nucleon volume is zero, when calculated on the nucleon surface where the gradient of the vacuum index is zero $[11,15]$.

Finally, we want to observe that our model could be reformulated in the framework of position dependent Schrodinger equation [23] and a possible link could be given by the following equation

$$
\phi_{\text {Schrodinger }}(m(r))=\int_{n}^{1} \phi\left(x-\frac{c}{n^{\prime}} t\right) \rho\left(n^{\prime}\right) d n^{\prime}
$$

with $m(r)$ the position dependent mass defined by the following equation

$$
E_{\text {Nucleon }}(\tau)=m(r) c^{2}
$$

and $\rho\left(n^{\prime}\right)$ a probability distribution to be determined by the wave functions inserted in the equation (12).

\section{Conclusion}

We exposed our proposal of a deterministic microscopic model of internucleon forces which is a non-Hamiltonian time asymmetric generalization of a previous author model concerning nuclear reactions. We introduce a nuclear scalar electromagnetic potential which is coupled in a selfconsistent way to a hidden nuclear vacuum index, and we use them to describe with a path dependent force nucleon dynamics, exploiting an analogy with Bjerknes acoustic forces. Our proposal doesn't use conventional symmetries and quantum numbers of standard approach since we think they are emergent properties of hidden electrodynamic forces. In particular we try to describe stability of compound nuclei by a dynamic equilibrium of non-instantaneous repulsive and attractive hidden forces.

We want to remark that since nuclear reactions concerns unobservable cinematic properties of individual accelerated pulsating nucleons (and neither their interaction potentials nor the electromagnetic radiation emitted by them are observable), it is mandatory to look for alternative formulations of classical mechanics and electrodynamics of extended deformable objects, such as the old Weber electrodynamics and Hertzian electrodynamics (which use convective time derivative instead of partial one), or the recent new formulation of Mashhoon.

We think that this search program could offer a completely different approach to test the validity of Quantum Mechanics, independently from Bell inequalities, since both modern theoretically models either experiments show the incompleteness of the Copenhagen standard approach and of Born probabilistic interpretation of quantum wave functions to describe the accelerated dynamics of extended pulsating nucleons. Finally we hope that our model will encourage and stimulate new experiments searching for time dependent nuclear binding energies and delayed emission of neutrons and electromagnetic radiations.

\section{References}

1. Hahn O, Strassmann F (1939) Über den Nachweis und das Verhalten der bei der Bestrahlung des Urans mittels Neutronen entstehenden ErdalKalimetalle. Naturwissenschaften 27: 11-15.

2. Meitner L, Frisch OR (1939) Disintegration of Uranium by Neutrons: a New Type of Nuclear Reaction. Nature (London) 143: 239-240.

3. Bohr N, Wheeler JA (1939) The Mechanism of Nuclear 
Fission. Phys Rev 56(5): 426.

4. Von Neumann J (1932) Mathematical Foundations of Quanatum Mechanics. Princeton University Press, USA.

5. Bulgac A, Jin S, Stetcu I (2020) Nuclear Fission Dynamics: Past, Present, Needs and Future. Front Phys 8: 63.

6. Bulgac A (2020) Fission-Fragment Excitation Energy Sharing Beyond Scission. Phys Rev C 102(4): 044609.

7. Randrup J, Vogt R (2021) Angular Momentum Effects in Fission. Phys Rev C 103(1): 014610.

8. Binesh Z (2020) Static Properties of Neutron-Rich and Proton-Rich Isotopes. Phys Sci \& Biophys J 4(2): 1-5.

9. Gainutdinov RKh, Mutygullina AA (2002) NonHamiltonian Nature of Low Energy Nucleon Dynamics in an effective Field Theory. Quantum Physics.

10. Gamow G (1928) Zur Quantentheorie des Atomkernes. Phys Z 51: 204-212.

11. Bei G (2021) A Hidden Variable Model of Unstable Quantum States. Phys Sci \& Biophys J 5(1): 1-3.

12. Dürr D, Grummt R, Kolb M (2011) On the Time-Dependent Analysis of Gamow Decay. Eur J Phys 32: 1435.

13. Bush JWM, Durey M (2020) Hydrodynamic Quantum Field Theory: The Onset of Particle Motion and the Form of the Pilot Wave. Front Phys 8: 300.

14. Bei G, Passaro D (2020) Symmetry Breaking Model of Volume Pulsating Walking Droplets. Progress in Physics
16(2): 102-105.

15. Bei G (2021) A Hidden variable model of Nuclear Reactions. IJAP 8(2): 1-4.

16. White H, Bailey P, Lawrence J, George J, Vera J (2020) A discussion on a dynamic vacuum model: Derivation of Helmholtz equation from Schrodinger equation. Physics Open 1: 100009 .

17. Hooft G (2021) Fast Vacuum Fluctuations and the Emergence of Quantum Mechanics. Quantum Physics 27.

18. Suzuki Y, Mertes KM (2019) Vacuum Texture: A New Interpretation of Quantum Mechanics and a New Loophole for Bell's Inequality Measurements that preserves Local Realism and Casuality. Quantum Physics.

19. Gill RD (2014) Statistics, causality and Bell's Theorem. Statist Sci 29(4): 512-528.

20. Hooft G (2021) Explicit construction of Local Hidden variables for any quantum theory up to any desired accuracy. Physics, pp: 1-15.

21. Bunchaft F, Carneiro S (1997) Weber-like interactions and energy conservation. Found Phys Lett 10: 393-401.

22. Mashhoon B (2020) Toward Nonlocal Electrodynamics of Accelerated Systems. Universe 6: 229.

23. Rego-Monteiro MA, Rodrigues LMCS, Curado EMF (2016) Position-dependent mass quantum Hamiltonians: General approach and duality. J Phys A: Math Theor 49: 125203. 